\title{
Генерация поверхностных плазмон-поляритонных волн в углеродной нанотрубке
}

\author{
И.О. Золотовский ${ }^{1}$, А.С. Кадочкин ${ }^{1}$, Ю.С. Дадоенкова ${ }^{1}$, С.Г. Моисеев ${ }^{1,2, *}$, \\ А.А. Фотиади ${ }^{1,3}$ \\ ${ }^{1}$ Ульяновский государственный университет \\ ${ }^{2}$ Ульяновский филиал Института радиотехники и электроники им. В.А. Котельникова РАН \\ ${ }^{3}$ Université de Mons, 20 Place du Parc, B7000 Mons, Belgium \\ *e-mail: serg-moiseev@yandex.ru
}

DOI:10.31868/RFL2018.40-41

В настоящей работе рассмотрена модель компактного генератора поверхностных плазмон-поляритонов (ППП), использующего оригинальный механизм усиления посредством прямой передачи энергии от носителей постоянного электрического тока (волны дрейфового тока) к электромагнитной волне (ППП волне). Подобный принцип хорошо известен в СВЧ технике (лампа бегущей волны и др.). Необходимым условием взаимодействия указанных волн является выполнение фазового синхронизма, то есть равенство фазовой скорости ППП волны и дрейфовой скорости носителей заряда. Основные положения предлагаемого подхода изложены в наших работах $[1,2]$, посвященных проблеме усиления ППП в полупроводниковой пленке с грефеновым слоем. В настоящей работе данный подход применен к отдельному наноразмерному объекту цилиндрической симметрии - углеродной нанотрубке (УНТ) [3]. Обратная положительная связь в УНТ реализуется за счет рассеяния усиленной ППП волны на периодическом потенциале, созданным профилированной поверхностью подложки.

УНТ длиной $L$ и радиусом $a$ расположена в вакууме (воздухе), разность электрических потенциалов приложена к еe концам. Рассматривается неизлучающая ТМ-мода, электрическое поле которой имеет ненулевую компоненту $E_{z}$ вдоль оси УНТ (оси координат $z$ ). Наличие радиальной составляющей поля необходимо для реализации взаимодействия электрического тока накачки и ППП волны. Распределение поля ППП волны и спектральные характеристики УНТ рассчитаны в рамках гидродинамической модели для электронного газа в УНТ с использованием уравнений Максвелла и стандартных граничных условий для электромагнитного поля [4].

Основное уравнение, описывающее взаимодействие поверхностной электромагнитной волны с дрейфовым током проводимости $I_{d}$, задаётся соотношением [5]

$$
\frac{d E_{z}}{d z}+i \frac{\omega}{V_{p h}} E_{z}=-\frac{1}{2}\left(\frac{\omega}{V_{p h}}\right)^{2} K I_{d},
$$

где $V_{p h}=\omega / \beta, \omega$ и $\beta$ - фазовая скорость, частота и постоянная распространения ППП, $K$ - параметр связи, определяющий эффективность взаимодействия тока и формирующейся поверхностной электромагнитной волны. Для описания взаимодействия токовой и ППП волн в условиях сильной связи, когда под влиянием поля электромагнитной волны амплитуда токовой волны становится модулированной по длине УНТ, уравнение (1) необходимо дополнить уравнением

$$
\frac{d^{2} \Delta I}{d z^{2}}+2 i \frac{\omega}{V_{0}} \frac{d \Delta I}{d z}-\frac{1}{V_{0}^{2}}\left(\omega^{2}-\omega_{q}^{2}\right) \Delta I=i \frac{\omega}{V_{0}} \frac{I_{d 0}}{2 U_{0}} E_{z}
$$


где $U_{0}-$ приложенная разность потенциалов, вызывающая постоянный ток $I_{d 0}, V_{0}$ - дрейфовая скорость носителей тока, $\omega_{q}$ - редуцированная плазменная частота, учитывающая влияние геометрических размеров волновода, $\Delta I(x)$ - малое возмущение тока. Совместное решение уравнений (1) и (2) позволяет определить коэффициент усиления ППП, распространяющегося в направлении дрейфа электронов тока накачки.

В приближении неистощимой токовой накачки динамика распространения прямой и обратной поверхностных волн в УНТ с периодическим потенциалом описывается системой уравнений для лазеров с распределенной обратной связью [6]. Для некоторых значений периода модуляции $\Lambda$ периодического потенциала в УНТ реализуется режим генерации поверхностных волн. Советующее уравнение принимает вид

$$
\left(\alpha / 2-i k_{0} \Delta n_{\text {eff }}\right) \operatorname{sh}(S L)-S \operatorname{ch}(S L)=0,
$$

где $\alpha$ - коэффициент усиления ППП, $S^{2}=|\kappa|^{2}+\left(\alpha / 2-i k_{0} \Delta n_{\text {eff }}\right)^{2}, \kappa \approx \chi k_{0} n_{\text {eff }} / 2$, $\chi<<1$ - глубина модуляции волнового числа ППП, $\Delta n_{\text {eff }}=n_{\text {eff }}-\pi m / \Lambda k_{0} \quad(m-$ целое число) - отстройка эффективного показателя преломления ППП.

На рис. 1 показан результат расчета частотной зависимости пропускания УНТ радиуса $a=5$ нм с дрейфовой скоростью электронов проводимости $5 \cdot 10^{7} \mathrm{~cm} / \mathrm{c}$. Можно видеть, что вблизи частот $1.24 \cdot 10^{13} \mathrm{c}^{-1}$ и $1.55 \cdot 10^{13} \mathrm{c}^{-1}$ выполняется условие генерации ППП в УНТ.

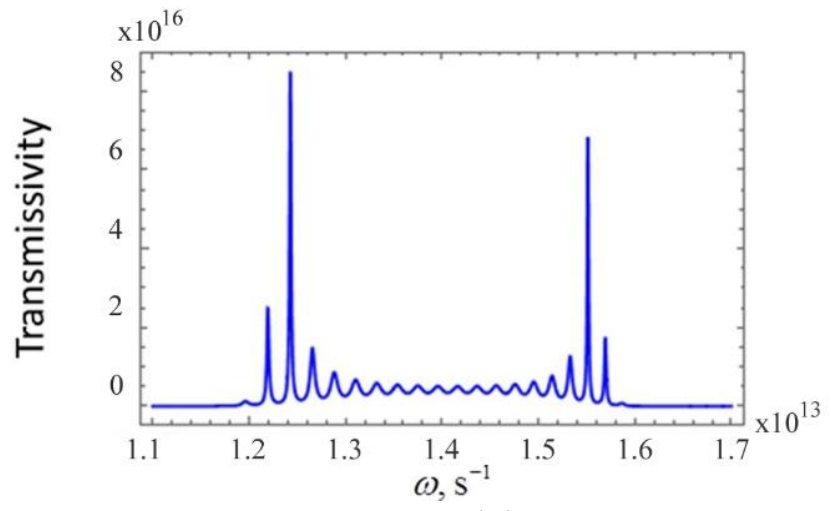

Рис. 1. Частотная зависимость коэффициента пропускания УНТ.

Работа выполнена при финансовой поддержке Министерства образования и науки РФ (проекты 3.7614.2017/9.10, 3.5698.2017/9.10, 3.3889.2017/4.6), Российского научного фонда (проект 18-12-00457) и Российского фонда фундаментальных исследований (проект 17-02-01382).

\section{Литература}

1. Y. S. Dadoenkova, S. G. Moiseev, A. S. Abramov, A. S. Kadochkin, A. A. Fotiadi, I. O. Zolotovskii, Annalen der Physik (Berlin) 529, 1700037 (2017)

2. I. O. Zolotovskii, Y. S. Dadoenkova, S. G. Moiseev, A. S. Kadochkin, V. V. Svetukhin, and A. A. Fotiadi, Phys. Rev. A 97, 053828 (2018)

3. A. S. Kadochkin, S. G. Moiseev, Y. S. Dadoenkova, V. V. Svetukhin, I. O. Zolotovskii, Optics Express 25, 27165-27171 (2017)

4. A. Moradi, Physics of Plasmas 21, 032106 (2014)

5. Д. И. Трубецков, А.Е. Храмов. Лекиии по СВЧ электронике для физиков. Том 1. (М: Физматлит, 2003)

6. A. Yariv, P. Yeh, Optical Waves in Crystals (Wiley: New York, 1984). 